\title{
Follow-up the Microbial Growth-down Rates of the Inflammatory Periodontal Pockets Using Radiotherapy, Taif Region, KSA
}

\author{
${ }^{*}$ Sherifa Mostafa M. Sabra \\ ${ }^{*}$ Const., Asst. Prof. Microbiology specialty, Micro. Br., Biology Dept., Science College, Taif University, KSA
}

\begin{abstract}
This research was carried out to follow-up the microbial growth-down rates of the inflammatory periodontal pockets using radiotherapy, Taif Region, KSA. The patients (Pts.) under study were (No. $=25$ pts.), they had chronic pockets periodontitis, they were divided into 20Pts. as study group (SG) and 5Pts. as control group (CG). SG had received radiotherapy (RT) and $C G$ had received irrigation with normal saline instead of $R T$. The proses period had taken (2monthes $=8$ weeks), that were divided into: stagel (baseline proses) at ( $1^{\text {st }}$ week), stage 2 (treatment proses) at $\left(2^{\text {nd }}, 3^{\text {rd }}, 4^{\text {th }}, 5^{\text {th }}\right.$ and $6^{\text {th }}$ week), and stage 3 (follow up proses) at $\left(7^{\text {th }}\right.$ and $8^{\text {th }}$ week). Microbial specimens were collected from periodontal pockets to detect microbial growth rates (MGRs). SG and CG were revealed mean microbial growth rates as $73.3 \%$ for both at stagel (baseline proses during $1^{\text {st }}$ week). $S G$ were $(53.3,46.7,33.3,20,13.3 \%)$ and $C G$ were $(60,53.3,40,33.3$ and $26.7 \%)$ at stage2 (treatment proses during $2^{\text {nd }}, 3^{\text {rd }}, 4^{\text {th }}, 5^{\text {th }}$ and $6^{\text {th }}$ week). SG were (6.7 and $\left.00 \%\right)$ and $C G$ were (20 and 13.3\%) at stage 3 (follow-up proses during $7^{\text {th }}$ and $8^{\text {th }}$ week) respectively. RT revealed anti-microbial effect and reduction of inflammation in periodontal pockets, also, supports healing of periodontal pockets through microbial eliminating.
\end{abstract}

Key words:Microbial Growth-down Rates, Inflammatory periodontal pockets, Patients (Pts.), Number (No.), Study group (SG), Control group (CG), Radiotherapy (RT), Microbial growth rates (MGRs).

\section{Introduction}

Periodontitis is a microbial related inflammatory disease Non-surgical treatment of such destructive disease was based on the elimination of MOs deposits adhered to tooth surfaces, primarily by means of scaling ${ }^{[1]}$. Since it is known that periodontal disease is a chronic inflammatory disease caused by MOs infection, the antimicrobial and detoxifying effect of RT is advantageous in periodontal therapy ${ }^{[2]}$. RT has bactericidal effectiveness ${ }^{[3]}$. Moreover, there is a significant suppression of Actinomycete mcomitans, an invasive bacterium that is associated with aggressive forms of periodontal disease. Actinomycete mcomitans is not only present on the diseased root surface, but it also invades the adjacent soft tissues, making it difficult to be removed by mechanical periodontal instrument alone ${ }^{[4]}$. RT provides a non-antibiotic solution. Actinomycete mcomitans has also been found in atherosclerotic plaques ${ }^{[5]}$. This makes it even more compelling to seek methods to control this aggressive MOs. RTs are very effective for soft tissue applications including incision, hemostasis and coagulation ${ }^{[6]}$. Most conventional methods used to treat the disease involve disruption of the biofilm by mechanical removal of sub-gingival plaque and, sometimes, the adjuvant use of anti-microbial agents and mechanical surgical debridement of pocket and root surfaces damaged as a result of periodontal disease. An alternative (ecological) approach would be to get rid of the putative $\mathrm{MOs}^{[7]}$. Non-surgical therapy leads to resolution of inflammation and reduction of MOs load ${ }^{[8]}$. These instruments help to reduce the MOs load by mechanical removal of plaque and calculus. Thus, surgical therapy performed in cases with persistent inflammation, deeper pockets, peri-pathogens persist in the mixed-spp., plaque biofilm on tooth surfaces, adhere to and enter the epithelial cells, and are tissue invasive in nature ${ }^{[9]}$. These are sources for re-colonization and reinfection. The limitations of the conventional therapy have probed to implement the use of adjunctive antimicrobial measures. RT periodontal therapy has attracted attention recently as a potential alternative or adjunct to conventional mechanical debridement ${ }^{[10]}$. RT, part of Laser energy scatters and penetrates during irradiation into periodontal pockets. The attenuated Laser at a low energy level might then stimulate the cells of surrounding tissue resulting in reduction of inflammatory conditions in cell proliferation, increased flow of lymph improving the periodontal tissue attachment and possibly reducing MOs load ${ }^{[11]}$. It is an excellent soft tissue surgical Laser, a antimicrobial effect ${ }^{[12]}$. Effect of RT on MOs by effect on microbial cell contents, it's also associated with dental caries and periodontitis ${ }^{[13]}$.

The aim: Follow-up the microbial growth-down rates of the inflammatory periodontal pockets using RT, Taif Region, KSA. Detection of MGRs with using RT as recent irradiation treatment, examine the effects of RT in the reducing MGRs during the treatment proses of chronic periodontitis. 


\section{Materials and Methods}

Patient groups preparation: This study was conducted at private dental clinics at Taif Region, that had done after gotten an agreements from dental clinic owners, dentists and patients. A total Pts. were (No.=25Pts.) with chronic periodontitis were selected, then were divided into 20Pts. study group (SG) and 5Pts. control group (CG), their ages (20-50yrs.).

Proses preparation: All Pts. were subjected to treatment, SG had received RT, CG had received irrigation with normal saline instead of RT. The process period had taken ( 2 monthes $=8$ weeks), that were divided into: stage 1 (baseline proses) at ( $1^{\text {st }}$ week), stage2 (treatment proses) at $\left(2^{\text {nd }}, 3^{\text {rd }}, 4^{\text {th }}, 5^{\text {th }}\right.$ and $6^{\text {th }}$ week), and stage 3 (follow up proses) at ( $7^{\text {th }}$ and $8^{\text {th }}$ week).

Microbial Specimens collection: Microbial specimens were collected aseptically with sterile paper tips from periodontal pockets to detect MGRs during the proses of use RT and SG and CG.

Microbial methods: Specimens were processed up to $24 \mathrm{hrs}$. after collection, were put into Patent Microbial Culture Petri (PMCP) dishes, were reading within 24-48hrs. of incubation at $37^{\circ} \mathrm{C}^{[14]}$.

Data Analysis:The data were recorded during the experiments and study periods were also entered into Microsoft Excel Sheet program for summarized and analyzed ${ }^{[15-16]}$.

\section{Results and discussion}

Table and graph1: Prevalence of the mean microbial growth rates during study stages of the proses

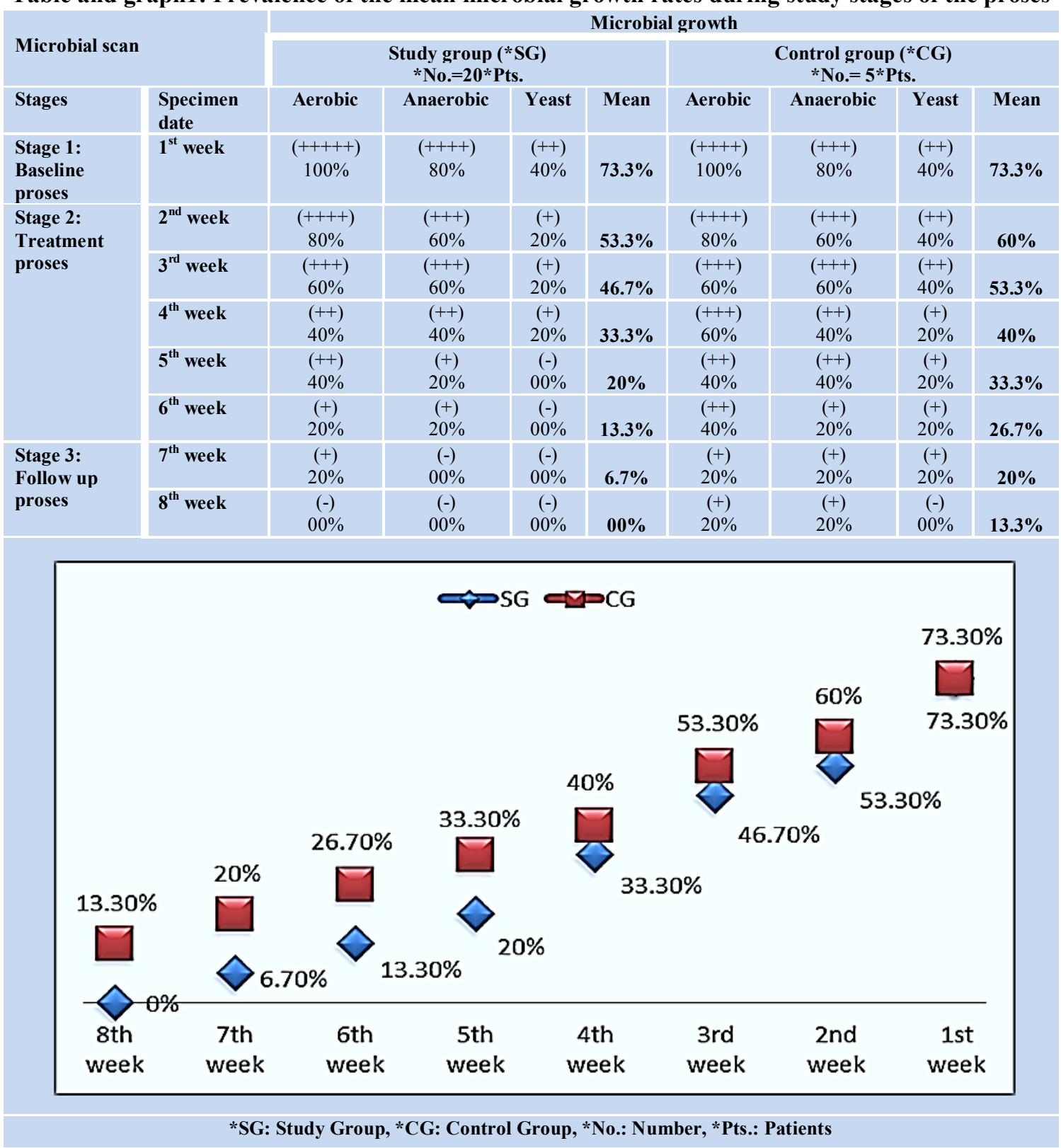


Table and graph1, showed prevalence of the mean microbial growth rates during study stages of the proses. SG and CG were revealed mean MGRs rates as $73.3 \%$ for both at stage1 (baseline proses during $1^{\text {st }}$ week). SG were $(53.3,46.7,33.3,20,13.3 \%)$ and CG were $(60,53.3,40,33.3$ and $26.7 \%)$ at stage2 (treatment proses during $2^{\text {nd }}, 3^{\text {rd }}, 4^{\text {th }}, 5^{\text {th }}$ and $6^{\text {th }}$ week). SG were $(6.7$ and $00 \%)$ and CG were $(20$ and $13.3 \%)$ at stage 3 (follow-up proses during $7^{\text {th }}$ and $8^{\text {th }}$ week) respectively. So the proses had done showed that, SG results were conformed the RT expressed as antimicrobial and it was more effective in decline the MOs growth rates in inflammed periodontal pockets and was enhancing the cure than CG results. Periodontitis is a microbial related inflammatory disease Non-surgical treatment of such destructive disease was based on the elimination of MOs deposits adhered to tooth surfaces, primarily by means of scaling ${ }^{[1]}$. The antimicrobial and detoxifying effect of RT is advantageous in periodontal therapy ${ }^{[2-3]}$. Moreover, there is a significant suppression of an invasive bacterium that is associated with aggressive forms of periodontal disease $\mathrm{e}^{[4-5]}$. This control this aggressive MOs, RTs are very effective for soft tissue ${ }^{[6]}$. An alternative RT approach would be to get rid of the putative $\mathrm{MOs}^{[7]}$. Non-surgical therapy leads to resolution of inflammation and reduction of MOs load ${ }^{[8]}$. RT periodontal therapy has attracted attention recently ${ }^{[10]}$. RT, part of Laser energy scatters and penetrates during irradiation into periodontal pockets, reducing MOs load ${ }^{[11]}$. It is an excellent soft tissue surgical Laser, a antimicrobial effect ${ }^{[12]}$. Effect of RT on MOs by effect on microbial cell contents, it's also associated with dental caries and periodontitis $^{[13]}$.

\section{Conclusions}

MGRs showed more declined with uses of RT to patients and it demonstrated a significant antimicrobial effect. Therefore, RT can form an integral part of periodontal therapy in the future. However, further evaluate the long-term effects of RT as microbiological scans. This effect on specific MOs and time taken for MOs recolonization needs to be determined by further studies.

\section{Acknowledgment}

Allot of grateful will be sending to all dental clinic owners, Dentists, and patients for their co-operation in the proses of research and producing this paper search. Also allot grateful will be sending to Micro. Lab. Staff for their help in this work.

\section{References}

[1]. Akoi, M., 2008. Current status of clinical laser applications in periodontal therapy, General Dentistry, PP: 674-687.

[2]. Moritz, G., 1997. Bacterial reduction in periodontal pockets through irradiation with a diode laser: a pilot study, J. Cline. Laser Med. Surg., 15: 33-37.

[3]. Harris, Y., 2004. Therapeutic ratio quantifies laser antisepsis: Ablation of Porphyromon as gingivalis with dental lasers, Lasers Surg. Med., 35: 206-213.

[4]. Ciancio, C., 2006. Wound healing of periodontal pockets using the diode laser, Applications of 810nm Diode Laser Technology: A Clinical Forum, PP: 14-17.

[5]. Fontana, K., 2004. Microbial reduction in periodontal pockets under exposition of a medium power diode laser: an experimental study in rats, Lasers Surg. Med., 35:263-268.

[6]. Ciancio, C., 2006. Effect of a diode laser on Actino bacillus Actinomycete mcomitans, Biological Therapies in Dentistry, 22:233239.

[7]. Spahr, K., 2006. Periodontal infections and coronary heart disease: role of periodontal bacteria and importance of total pathogen burden in the Coronary Event and Periodontal Disease (CORODONT), Study, Arch. Int. Med., 166:554-559.

[8]. Ishikawa, I., Aoki, A., Aristeo, A., Takasaki, Mizutani K., SasakiK, M. and Izumi, Y., 2009. Application of lasers in periodontics: true innovation or myth? Periodontal, 50:90-126.

[9]. Euzebio, V., Andrade, A., Toaliar, M., Conde, M., Zezell, M., CaiS, P. and Micheli, G., 2012. Clinical and microbiological evaluation of high intensity diode laser adjutant to non-surgical periodontal treatment: a 6-month clinical trial, Cline. Oral. Invest. DOI. 14:11-15.

[10]. Gokhale, S., Padhye, A., Byakod, G., Jain, S., Padbidri, V. and Shivaswamy, S., 2012. A Comparative Evaluation of the Efficacy of Diode Laser asan Adjunct to Mechanical Debridement Versus Conventional Mechanical Debridement in Periodontal Flap Surgery: A Clinical and Microbiological Study. Photo medicine and Laser Surgery,30:598-603.

[11]. Marsh, P., 1991. Sugar, fluoride, $\mathrm{pH}$ and microbial homeostasis in dental plaque. Proc. Finn. Dent. Soc., 87:515-525.

[12]. Haffajee, A. and Socransky, S., 1994. Microbial etiological agents of destructive periodontal diseases. Periodontal, 5:78-111.

[13]. Darveau, R.., Tanner, A. and Page, R., 1997. The microbial challenge in periodontitis. Periodontal, 14:12-32.

[14]. Murray, P., Baron, M. and Pfaller, F., 2007. Manual of Cline. Micro. $9^{\text {th }}$ Ed., American Society Micro., London.

[15]. Coulombier, D., Fagan, R., Hathcock, L. and Smith, C., 2001. Epi. Info. 6 Version 6.04.A Word Processing, Database and Statistical Program for Public Health. Centers for Disease Control and Prevention (CDC), Atlanta, Delaware, USA.

[16]. Mirabella, J., 2011. Statically analysis with SPSS/PASW, Anon statistians guide and tutorial, SPSS. Inc. 